\title{
Toxina botulínica: uma nova opção para o tratamento da neuralgia pós-herpética?
}

\author{
Botulinum toxin: a new option for the treatment \\ of post-herpetic neuralgia?
}

\section{Toxina botulínica: una nueva opción para el tratamiento de la neuralgia post herpética?}

\author{
Samantha Talarico*
}

\section{Resumo}

A dor neuropática pode ocorrer após injúria do sistema nervoso central ou periférico, gerando dor espontânea, alodínea e hiperalgesia. Uma das principais causas de dor neuropática é a neuralgia pós-herpética. Sua incidência aumenta em pacientes com idade mais avançada, o que dificulta ainda mais o tratamento, muitas vezes limitado por seus efeitos colaterais, principalmente cognitivos. A combinação de tratamentos é prática clínica comum e novas modalidades terapêuticas vêm sendo aplicadas para aliviar essa condição. A toxina botulínica tipo A tem sido usada para diversos quadros clínicos associados à hiperatividade muscular, como distonia cervical. Recentemente, foram propostos mecanismos analgésicos independentes da contração muscular, ainda pouco conhecidos.

Descritores: Neuralgia; Neuralgia pós-herpética; Toxinas botulínicas tipo A.

\begin{abstract}
Neuropathic pain can occur after injury to the peripheral and/or central nervous system, resulting in spontaneous pain, allodynia and hyperalgesia. One of the main causes of neuropathic pain is post-herpetic neuralgia. It occurs increasingly with age and that makes the treatment even more complicated due to the limitation caused by cognitive adverse effects. Combining therapies with different mechanisms of action is usual in clinical practice and new procedures are considered to treat this condition. Botulinum toxin type A has been used to treat certain disorders related to muscle overactivity. Not well known analgesic mechanisms have been proposed recently, independent of the muscle contraction.
\end{abstract}

Keywords: Neuralgia; Neuralgia, postherpetic; Botulinum toxins, type A.

\section{Resumen}

El dolor neuropático puede darse después del daño al sistema nervioso central o periférico, provocando dolor espontáneo alodinia y hiperalgesia. Una de las principales causas del dolor neuropático es la neuralgia postherpética. Su incidencia aumenta en pacientes de edad avanzada, lo que dificulta todavía más el tratamiento, muchas veces limitado por sus efectos colaterales, principalmente cognitivos. La combinación de tratamientos es práctica clínica común y se vienen aplicando nuevas modalidades terapeúticas para aliviar esta situación. La toxina botulínica tipo A ha sido usada en diversos cuadros clínicos asociados a la hiperactividad muscular, como distonía cervical. Recientemente, fueron propuestos mecanismos analgésicos independientes de la contracción muscular, todavía poco conocidos.

Palabras clave: Neuralgia; Neuralgia posherpética; Toxinas botulínicas tipo A. 


\section{Introdução}

O herpes-zóster agudo acontece a partir da reativação do vírus varicela zoster, que se encontra latente no gânglio dorsal. ${ }^{1}$

Os gatilhos que levam a essa reativação ainda não foram bem elucidados, mas parecem consequentes a múltiplos fatores, envolvendo a relação entre o vírus e hospedeiro.

Ocorre particularmente em pacientes com queda da imunidade celular, de causa conhecida ou não, associada a uso de medicações, doenças ou idade avançada. ${ }^{2,3}$

$\mathrm{O}$ vírus então reativado sofre replicação $\mathrm{e}$ se propaga pelo nervo afetado, bem como pela corrente sanguínea, ativando o sistema imune e induzindo inflamação, podendo lesar neurônios periféricos e centrais. Atinge o gânglio dorsal, adjacente à medula espinhal, onde estabelece latência.

Manifesta-se com dor aguda, que dura de duas a quatro semanas, seguida durante sete a dez dias por rash eritematoso maculopapular, que evolui para vesículas, em geral restringindo-se a um dermátomo tipicamente unilateral e não costumando ultrapassar a linha média. ${ }^{2}$

Em indivíduos imunocomprometidos, embora possa apresentar-se inicialmente da forma habitual, o rash tende a ser mais grave e de duração prolongada, podendo se estender a grandes áreas. ${ }^{2}$

A neuralgia pós-herpética (NPH), principal complicação do herpes-zóster, ${ }^{3,4,5}$ consiste na persistência da dor por período superior a quatro meses após o início do desenvolvimento do rash cutâneo e ocorre em cerca de $10 \%$ dos pacientes. Tem sua incidência aumentada em pacientes com idade avançada ${ }^{1,2,4}$ na localização oftálmica da lesão de herpes-zóster.

Mais da metade dos pacientes com NPH apresenta distúrbios do sono, diminuição de atividades diárias, do apetite e da libido, além de comprometimento significativo da sua vida social, com importante diminuição da qualidade de vida. O quadro pode evoluir para dor crônica, muitas vezes debilitante, havendo inclusive relatos de tentativas de suicídio em casos mais graves. ${ }^{2,3,4}$

O mecanismo fisiopatológico da neuralgia envolve os neurônios aferentes (nociceptores), terminações nervosas especializadas que respondem a estímulos (térmicos, químicos ou mecânicos) de produção de dor. Através das fibras curtas (fibras C e A- $\partial$ ), os nociceptores ativados são capazes de traduzir o estímulo recebido em estímulo elétrico e transmiti-lo do sistema nervoso periférico para o sistema nervoso central (SNC), onde será interpretado no córtex como dor. ${ }^{6,7,8}$

No entanto, o papel das fibras curtas não se restringe à sinalização. Frente a uma lesão, secretam neuropeptídeos como o peptídeo relacionado ao gene da calcitonina (CGRP) e à substância P, recrutam células imunológicas e induzem vasodilatação. ${ }^{9}$ Estes neurotransmissores contribuem para a manutenção do processo inflamatório e iniciam o processo de sensibilização periférica, que culmina com a exacerbação da resposta ao estímulo doloroso. ${ }^{8}$

Para gerar resposta aos estímulos, os neurônios aferentes utilizam vários receptores, transmissores, canais iônicos transdutores e moduladores. O receptor valinoide de potencial receptor transiente (TRVP1), ativado pela capsaicina, atua como marcador molecular da nocicepção. ${ }^{10}$ Sofre alterações induzidas pelas prostaglandinas pela e bradicinina, reduzindo o tempo pós-hiperpolarização da membrana neural, causando redução do limiar para disparo da fibra nervosa. ${ }^{8}$

$\mathrm{Na} \mathrm{NPH}$, a inflamação na raiz do gânglio dorsal leva a alterações celulares e de conexão da via nociceptiva do SNC, seguido de deaferentação anatômica."

Trata-se de uma dor neuropática, definida pela Associação Internacional de Estudos da Dor como: dor iniciada ou causada por uma lesão primária ou disfunção do sistema nervoso; ou ainda dor causada por lesão do sistema nervoso central ou periférico (ou ambos), manifestando-se por sinais e sintomas sensoriais..$^{10,12}$ Decorre do mau funcionamento do sistema nervoso, não dependendo do estímulo contínuo de lesão tecidual. ${ }^{13}$

Após uma lesão do SNC ou periférico, na tentativa de reparar o dano, ocorrem anormalidades da transmissão ${ }^{6}$ e há uma perda da inibição dos sinais locais da dor nociceptiva, com o prejuízo aos nervos periféricos; desenvolve-se um limiar mais baixo para ativação da dor nociceptiva, além da produção de descargas espontâneas e ectópicas, levando à sensibilização periférica. ${ }^{9}$ A atividade aumentada dos nervos periféricos e a inflamação levam a uma resposta alterada dos neurônios dorsais e consequente sensibilização 
central, que corresponde à hiperexcitabilidade dos neurônios nociceptivos centrais e modificações diretas em suas propriedades eletrofisiológicas. Aminoácidos excitatórios, como o glutamato, têm papel essencial neste processo., ${ }^{712}$

Após a morte de neurônios periféricos ocorre um rearranjo anormal dos neurônios do cordão espinhal deferente, que, mais facilmente excitável, pode gerar dor persistente e/ou espontânea (independente de estímulo ou dano tecidual).,13

Entre os sinais e/ou sintomas possivelmente observados destacamos: 1) deaferentação, que consiste em dor contínua secundária à injúria de nervo periférico, levando à perda ou desligamento das aferências ao SNC; 2) hiperalgesia, sensação de dor mais intensa que o usual; 3) hiperestesia, qualquer estímulo sensorial percebido de forma exagerada; 4) anestesia e perda sensorial profunda na área dolorida $;^{13}$ e 5) alodínea, dor com estímulo normalmente não doloroso, que pode estar relacionada à formação de novas conexões envolvendo os neurônios de transmissão centrais da dor.

A complexa fisiopatologia da NPH possibilita múltiplos alvos terapêuticos, mesmo que o mecanismo de ação que promova a analgesia ainda não seja completamente entendido. Assim, a resposta ao tratamento é imprevisível, e dois pacientes com neuralgia pós-herpética podem responder de forma diferente ao mesmo tratamento.

Até os dias de hoje, a prevenção destes quadros implica no diagnóstico precoce do herpeszóster e no início rápido do uso de antivirais. ${ }^{4}$

Embora a vacinação para herpes-zóster não seja citada nos guias de conduta atuais, parece apresentar certa eficácia na prevenção da NPH a curto prazo. Introduzida nos Estados Unidos em 2006, Zostavax ${ }^{\circledR}$ consiste numa vacina de vírus vivo atenuado que se mostrou segura e efetiva e que chegou recentemente ao Brasil. Trata-se, no entanto, de uma vacina ainda recente e, de acordo com revisão da Biblioteca Cochrane, não existem, até o momento, dados que suportem sua eficácia na prevenção de NPH a longo prazo, sendo necessários mais estudos, com um maior período de acompanhamento.

Levando em conta ainda o custo-benefício deste tipo de medida, o centro de controle de doenças e prevenção ${ }^{2}$ recomenda a vacinação apenas para indivíduos maiores de 60 anos., ${ }^{1,2}$
Os corticoides orais não parecem abreviar nem prevenir evolução para NPH., ${ }^{1,2}$

Consideradas, atualmente, como drogas de primeira linha pela maioria dos guias de conduta, os antidepressivos tricíclicos, como amitriptilina e desipramina, são largamente aplicados para o tratamento de dores neuropáticas. Embora bastante eficazes, apresentam importantes efeitos colaterais, principalmente colinérgicos, como boca seca, constipação e arritmias.,9

Anticonvulsivantes, como a gabapentina e a pregabalina, provavelmente reduzem os fenômenos de sensibilização central, por sua ação sobre a subunidade $\alpha 2 \delta$ - 1 do complexo do canal de $\mathrm{Ca}^{2+}$ voltagem-dependente presente nos neurônios. Estes têm como efeitos indesejáveis vertigem, sonolência, fadiga e boca seca.

Como tratamento tópico, são citados patch de lidocaína 5\%, com boa eficácia na redução da dor, inclusive mantida a longo prazo., ${ }^{1,4}$ Alguns autores argumentam que a eficácia deste método ainda não está tão bem-estabelecida, mas concordam que sua segurança de aplicação, sobretudo em idosos, justificaria seu uso. ${ }^{9}$ Outra opção disponível é o creme de capsaicina $0,075 \%$, com o inconveniente de liberar baixas quantidades de capsaicina, necessitando de aplicação de três a quatro vezes ao dia. ${ }^{1,4}$

Os opioides e tramadol são considerados por muitos terapia de segunda linha, talvez pelos riscos de seu uso em longo curso, como hipogonadismo, alterações da imunidade e dependência.

$\mathrm{O}$ uso dessas drogas, em geral, é limitado pela tolerância do paciente e pelos efeitos colaterais, e depende do contexto somático e psiquiátrico deste.,14 A combinação de diferentes regimes terapêuticos é bastante comum na prática, uma vez que nenhuma terapia isolada parece ser efetiva. Acredita-se que o uso de duas ou mais terapias, com mecanismos diferentes ou complementares, possibilite resultados mais efetivos no controle da dor. Isso deve sempre ser considerado em pacientes não responsivos à monoterapia de primeira linha, sendo recomendada a associação de dois tratamentos orais ou tratamento oral com tópico.

Além disso, as drogas geralmente utilizadas para controle da dor, a longo prazo, muitas vezes têm efeitos colaterais cognitivos, fato particularmente relevante no grupo afetado por esta 
condição, de faixa etária avançada e com múltiplas comorbidades. ${ }^{6,3}$

Como se trata de uma doença localizada, alguns autores sugerem que o início do tratamento seja feito com agentes tópicos, menos susceptíveis a efeitos colaterais e, caso necessário, acrescentado agente oral. ${ }^{1}$

Tendo em vista estes impasses para o controle da dor neuropática crônica, inúmeros tratamentos não farmacológicos vêm sendo propostos. ${ }^{9}$ Entre eles podem ser citados: 1) bloqueio simpático com anestésicos, que apresenta alívio da dor a curto prazo, acompanhado, muitas vezes, de bradicardia e hipotensão; 2) injeções epidurais de esteroides com resposta satisfatória, mas duração máxima de um mês; 3) estimulação da medula espinhal que, apesar de cirurgicamente invasiva, pode ser utilizada em pacientes sensíveis a múltiplas medicações ou com comorbidades de difícil manejo.'

Excisão da área dolorosa de pele mostrou-se ineficaz, com recorrência da dor, muitas vezes em maior intensidade do que antes do procedimento.

\section{Uso da toxina botulínica na neuralgia pós-herpética}

A toxina botulínica aparece como uma ótima opção, minimamente invasiva, segura, bem tolerada e realizada em consultório, que tem demonstrado redução da dor na NPH em alguns casos, ${ }^{7}$ embora seu mecanismo de ação ainda seja pouco claro.

O uso da toxina botulínica é particularmente interessante, pois parece não afetar apenas os neurônios colinérgicos, como se sabia anteriormente, mas também neurônios não colinérgicos e outras estruturas. Por exemplo, seu efeito sobre o sistema nervoso autônomo justifica seu uso para hiperidrose e sialorreia. Recentemente as propriedades analgésicas têm sido estudadas em diferentes situações. ${ }^{13}$

A toxina botulínica é uma exotoxina, produzida pela cultura do Clostridium botulinum, bactéria anaeróbia gram-positiva. São oito subtipos distintos de toxinas (A, B, C1, C2, D, E, F, G), sendo a mais potente e mais utilizada a do tipo A (BTX-A). A porção biologicamente ativa é composta por duas cadeias: a cadeia leve de 50 kD, responsável por sua ação tóxica; e a pesada, de 100 kD, responsável pela fixação da toxina ao neurônio, unidas por uma ponte dissulfídica. . $^{15,16,17}$

A toxina se fixa nas terminações nervosas no receptor proteico de membrana (SV2 no caso da BTX-A). Essa etapa é crucial para a atividade terapêutica, e é importante saber que ela aumenta de acordo com o grau de excitação da terminação nervosa.

A BTX-A é, então, internalizada por endocitose. A cadeia leve vai exercer uma atividade proteolítica sobre o complexo SNARE, indispensável para a fusão de vesículas de acetilcolina com a membrana pré-sináptica. ${ }^{4,18,16}$

A toxina, de acordo com seu sorotipo, vai quebrar uma ou outra proteína deste complexo (no caso da BTX-A a SNAP 25), e tem como consequência a inibição da translocação, exocitose e liberação de acetilcolina na fenda sináptica. ${ }^{16,18}$

Inicialmente, acreditava-se que as propriedades analgésicas da BTX-A estavam associadas à diminuição da contração muscular e provavelmente associada a suas propriedades colinérgicas.

Observou-se no tratamento de distonia cervical uma melhora significativa da dor, que precedia os efeitos miorrelaxantes, sendo mais duradouros e mais intensos do que se esperaria apenas como consequência do relaxamento muscular, sugerindo que houvesse uma ação analgésica independente. .6,19-21 $^{-12}$

Outra observação clínica foi de que pacientes que tinham as rugas frontais tratadas com BTX-A referiram clara melhora de migrânia, doença na qual a hipertonia muscular não desempenha qualquer papel.

Numerosos estudos experimentais em animais sugerem que as ações da toxina botulínica vão além da inibição motora, incluindo o processamento nociceptivo aferente. . $^{13,20}$

Em outro estudo, a BTX-A mostrou-se capaz de reduzir a liberação de substância $P$ em culturas embrionárias de neurônios da raiz dorsal de ratos. Os neurônios mostraram-se ainda mais sensíveis à BTX-A em comparação aos demais tipos de toxinas, demonstrando provável diferença em termos de afinidade de receptores dos diferentes tipos de toxinas. ${ }^{13}$ Este estudo mostrou efeito prolongado (15 dias) da BTX-A na liberação mediada por cálcio de substância P.

Por um lado a toxina inibe a liberação de acetilcolina particularmente no nível das terminações 
colinérgicas glandulares, o que explica sua eficácia na hiperidrose, hipersalivação e hiperlacrimejamento. Por outro lado, a toxina botulínica parece ser capaz de agir em múltiplos neuropeptídeos, como o glutamato, um aminoácido excitatório envolvido na sensibilização dos neurônios centrais e periféricos, a substância P e a CGRP, que contribuem com a vasodilatação, liberação de histamina; e, finalmente, sensibilização periférica durante a inflamação neurogênica. ${ }^{13,4}$

Estímulos nocivos fazem com que os tecidos periféricos liberem bradicinina, 5-hidroxitriptofano (5-HT) e prostaglandina, levando à ativação do primeiro neurônio aferente periférico. Nos aferentes terminais primários, glutamato, outros aminoácidos e peptídeos neuroativos são liberados e posteriormente propagam sensibilização central e ativação antidrômica.

A ativação antidrômica libera ainda mais glutamato, CGRP e substância P perifericamente e ainda causa a translocação do TRPV1, canal iônico, receptor da capsaicina na membrana neuronal aferente, aumentando a excitabilidade do nociceptor. Trata-se de um receptor calciopermeável, integrador de estímulos nocivos, que permite resposta ao calor e alterações do $\mathrm{pH}$, aumentando a excitabilidade do nociceptor.

CGRP e substância P funcionam como gatilho para a ativação mastocitária e subsequente liberação de histamina, vasodilatação e mais inflamação neurogênica, que contribui para uma ativação secundária.

Estudos em animais sugerem que a BTX-A interfere com a sensibilização periférica ao prevenir a liberação mediada por vesículas de substância $P$, glutamato e CGRP, assim como inibir a translocação do receptor TRPV1 (que, em situações de dor crônica, encontra-se anormalmente aumentada).

Em resumo, postula-se que BTX-A exerça seus efeitos na transmissão nociceptiva sensibilizada (anormal) por meio de quatro mecanismos similares, todos mediados por vesículas, pela inibição de proteínas SNARE e consequente bloqueio da exocitose: $:^{13}$

1. inibindo a liberação de glutamato nos tecidos periféricos;

2. reduzindo a liberação CGRP nos tecidos periféricos;

3. reduzindo a liberação tecidual de substância P;
4. reduzindo o tráfego de TRPV1 na membrana celular do neurônio periférico.

Desta forma, podemos levantar a hipótese de que a aplicação de toxina botulínica na zona dolorosa poderia reduzir a inflamação neurogênica, a sensibilização periférica e, consequentemente, a dor resultante. ${ }^{16}$

De acordo com revisão recente, o uso de toxina botulínica para NPH com alodínea foi considerado nível $\mathrm{B}$ de evidência (provavelmente efetivo e devendo ser considerado como opção terapêutica). ${ }^{17}$

Diversos estudos entre relatos de casos e ensaios clínicos têm sido publicados mostrando os efeitos destas aplicações e presumindo os reais mecanismos de ação desta terapêutica.

Os relatos de caso encontrados na literatura $^{11,22,23}$ utilizam como parâmetro de avaliação da dor a visual analog scale (VAS), que consiste em uma escala visual na qual o paciente deve assinalar a intensidade da dor sentida nas últimas 24 horas, com zero correspondendo à ausência de dor e dez à dor máxima sentida.

Em todos os casos, houve redução da dor com duração variando de 52 a 64 dias.

Em um dos casos relatados, a regressão da dor foi completa e se manteve por oito semanas. Os autores realizaram aplicações seriadas com intervalos de três meses e mantiveram assim o escore da dor próximo a zero. ${ }^{22}$

Existem, até os dias de hoje, cerca de 20 publicações que abordam o uso de BTX-A para dores neuropáticas. Destes, apenas cinco têm como causa a neuralgia pós-herpética. ${ }^{18}$

Klein AW, ${ }^{21}$ em 2004, foi pioneiro em chamar atenção para os efeitos analgésicos independentes da toxina botulínica. Relatou quatro casos de seu uso para condições dolorosas, sendo um deles de neuralgia pós-herpética. Foram utilizadas $20 \mathrm{UI}$ na área afetada com melhora da dor mantida por quatro meses. No entanto, não foram descritos método de aplicação, diluição ou marca da toxina utilizada.

No segundo relato da literatura dermatológica, Sotiriou e colaboradores ${ }^{11}$ relatam quatro casos de neuralgia pós-herpética tratados com BTX-A (Botox ${ }^{\circledR}$ na diluição $100 \mathrm{Ul}: 2 \mathrm{ml} \mathrm{NaCl}$ ), sendo aplicadas 5 UI por ponto, num total de 100 UI. A gravidade da dor foi avaliada por meio da 
VAS, com redução importante a partir do sétimo dia e manutenção até 64 dias. Na recidiva, a dor foi considerada de menor intensidade e tolerável pelos pacientes.

Posteriormente, foram realizados três ensaios clínicos, randomizados, duplos-cegos, placebocontrolados ${ }^{3,4,20}$ com o objetivo de avaliar o efeito analgésico direto de uma única aplicação de BTX-A nesta condição.

Em todos os estudos os resultados do grupo de toxina botulínica foram superiores aos dos grupos controle, com a melhora da dor ocorrendo nas duas primeiras semanas e mantendo-se estável 12 a 16 semanas e, em alguns casos, permanecendo com algum efeito até 24 semanas. ${ }^{3,4,20}$

\section{Técnica de aplicação}

Após demarcação da área de alodínea realizada através do tato, ${ }^{3}$ são determinados pontos equidistantes para a aplicação de BTX-A.

As técnicas de aplicação variaram quanto à disposição na área afetada, ora sendo aplicadas em linhas quadriculadas, ${ }^{3,4,11,20}$ ora dispostas em leque. ${ }^{24} \mathrm{Na}$ maioria dos estudos, BTX-A foi administrada por via subcutânea ou intradérmica com doses fracionadas variando de 2,5 a 7,5 UI/ $\mathrm{cm}^{2}$ na área dolorosa, não sendo ultrapassadas 200 UI no total. ${ }^{3,18}$

Em teoria, a toxina botulínica tem potenciais vantagens quando comparada a analgésicos orais, seja por sua longa duração de ação (aproximadamente três meses), pela facilidade de ser administrada diretamente no ponto doloroso, por sua excelente tolerabilidade, seja por seu perfil de segurança e ausência de efeitos sistêmicos. ${ }^{25}$

\section{Conclusão}

A BTX-A pode ser considerada uma opção na terapêutica da dor neuropática crônica, particularmente na NPH, condição capaz de comprometer amplamente a qualidade de vida dos pacientes e na qual os tratamentos atuais são pouco satisfatórios além de, muitas vezes, limitados por efeitos colaterais graves.

Embora promissora, por se tratar de uma nova modalidade terapêutica, necessitamos de novos estudos para que possa ser estabelecido um protocolo de diluições, métodos de aplicação e doses. Claro que, como em qualquer outra indicação da toxina botulínica, deve-se procurar avaliar a necessidade própria de cada caso.

\section{Referências}

1. Argoff CE. Review of current guidelines on the care of postherpetic neuralgia. Postgrad Med. 2011;123(5):134-42.

2. Harpaz R. Centers for Disease Control and Prevention. [Internet]. Atlanta: Division of Viral Diseases, National Center for immunization and Respiratory Diseases; [Atualizado em 2008, junho, 6]. Disponível em: http://www.cdc.gov/mmwr

3. Xiao L, Mackey S, Hui H, Xong D, Zhang Q, Zhang D. Subcutaneous injection of Botulinum Toxin A Is Beneficial in postherpetic Neuralgia. Pain Med. 2010;11(12):1827-33.

4. Apalla Z, Sotitiou E, Lallas A, Lazaridou E, loannides D. Botulinum Toxin A in Postherpetic Neuralgia: A parallel, Randomized, Double-Blind, Single-Dose, Placebo-controlled Trial. Clin J Pain. 2013;29(10):85764. doi: 10.1097/AJP.0b013e31827a72d2

5. Glogau RG. Review of the use or botulinum toxin for hyperhidrosis and cosmetic purposes. Clin J Pain. 2002;18(6 Suppl):S191-7.

6. Emad MR, Emad M, Taheri P. The efficacy of intradermal injectiopn of toxin in patients with post-herpetic neuralgia. Iran Red Crescent Med J. 2011;13(5):323-7

7. Argoff CE. A focused review on the use of botulinum toxins for neuropathic pain. Clin J Pain. 2002;18(6 Suppl):S177-181.

8. Rocha APC, Kraychete DC, Lemonica L, Carvalho LR, Barros GAM, Garcia JBS, et al. Dor: Aspectos Atuais da Sensibilização Periférica e Central. Revista Brasileira de Anestesiologia, 2007;57(1):95-105.

9. Sotiriou E, Apalla Z, Panagiotidou D, loannidis D. Severe post-herpetic neuralgia successfully treated with botulinum toxin A: three case reports. Acta Derm Venereol. 2009;89(2):214-5.

10. Oaklander AL. Chronic pain. ACP Medicine. 2011;1-19.

11. Sugeng MW, Yosipovitch G, Leok GC. Post herpetic neuralgia and the dermatologist. Int J Dermatol. 2001;40(1):6-11.

12. Current diagnóstico e tratamento - dor. Von Roenn JH, Paice JA, Preodor ME (ed.); Cosendey CHA, Setúbal S (trad.). Rio de Janeiro: McGraw-Hill Interamericana do Brasil, 2008. 360p.

13. Francisco GE, Tan H, Green M. Do botulinum toxins have a role in the management of neuropathic pain? A focused review. Am J Phys Med Rehabil. 2012;91(10):899-909.

14. Attal N. Therapeutic advances in pharmaceutical treatment of neuropatic pain. Rev Neurol (Paris). 2011;167(12):930-7. 
15. Azulay RD. Dermatologia. 6a edição. Rio de Janeiro: Guanabara Koogan, 2013.

16. Ranoux D. Botulinum toxin and painful peripheral neuropathies: what should be expected? Rev Neurol (Paris). 2011;167(1):46-50.

17. Jabbari B, Machado D. Treatment of refractory pain with botulinum toxinan evidence based review. Pain Med. 2011;12(11):1594-606.

18. Fabregat G, De Andrés J, Villanueva-Pérez VL, Asensio-Samper JM. Subcutaneous and perineural botulinum toxin type A for neuropathic pain: a descriptive review. Clin J Pain. 2013;29(11):1006-12.

19. Casale R, Tugnoli V. Botulinum toxin for pain. Drugs R D. 2008;9(1):11-27.

20. Ranoux D, Attal N, Morain F, Bouhassira D. Botulinum toxin type A induces direct analgesic effects in chronic neuropathic pain. Ann Neurol.

Recebido: 30/04/2014.

Revisado: 02/07/2014.

Aprovado: 31/07/2014.
2008;64(3):274-83.

21. Klein AW. The therapeutic potential of botulinum toxin. Dermatol Surg. 2004;30(3):425-5.

22. Ruiz HC, Bermejo P. Botulinum toxin type A in the treatment of neuropathic pain in a case of postherpetic neuralgia. Neurologia. 2008;23(4):259-62.

23. Liu HT, Tsai SK, Kao MC, Hu JS. Botulinum toxin A relieved neurophatic pai in case of post-herpetic neuralgia. Pain Med. 2006;7(1):89-91.

24. Ferreira JJ, Couto M, Costa J, Coelho M, Rosa MM, Sampaio C. Botulinum toxin for the treatment of pain syndromes. x Acta Reumatol Port. 2006;31(1):49-62.

25. Talarico S, Nascimento MM, Macedo F, Pecora C. A double-blind, randomized, comparative study between two type A botulinum toxin in the treatment of primary axillary hyperhidrosis. Dermatol Surg. 2007;33(S1):S44-S55.

\section{Samantha Talarico}

Instituto de Dermatologia Professor Rubem David Azulay. Santa Casa da Misericórdia. Rio de Janeiro, RJ, Brasil. 